\title{
04 | El legado del Movimiento Moderno. Conjuntos de vivienda masiva en ciudades europeas del Oeste y del Este. No tan diferentes...
}

\author{
_Javier Monclús y Carmen Díez
}

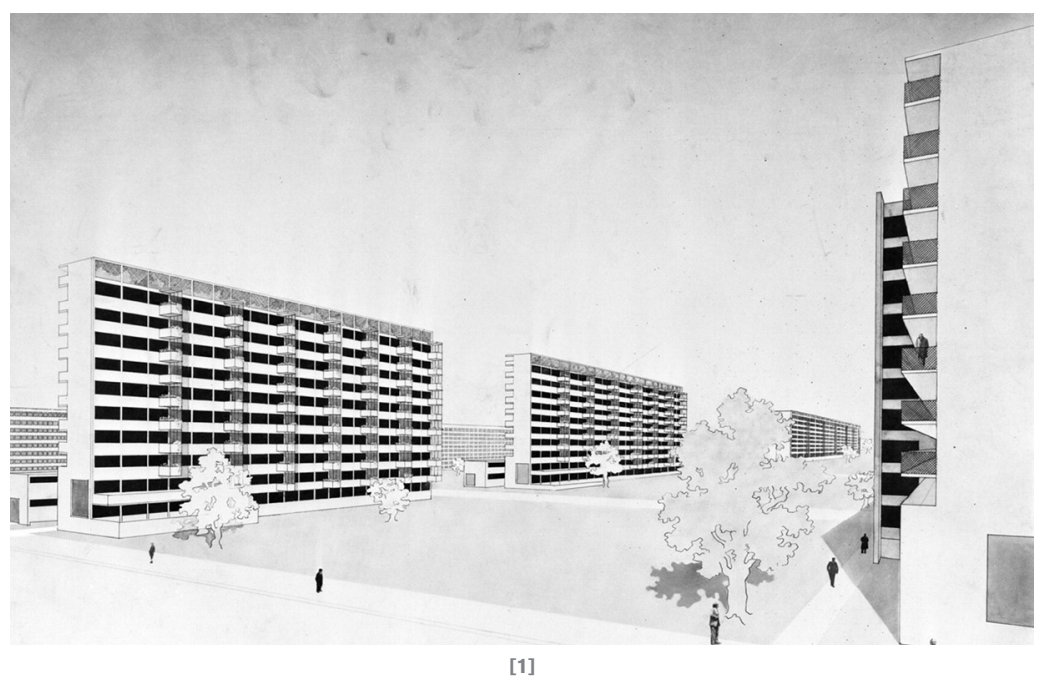

[1] W. Gropius, High-rise housing, CIAM 4, Bruxels, 1930. "Houses, Walk-ups or High-rise Apartment ros Blocks?" Bloques lineales modernos con grandes espacios abiertos como alternativa a los viejos modelos de vivienda y a to for de viva un a las formas urbanas tradicionales. Fuente: CIAM, Rationelle Bebauungsweisen, "Flach-, Mittel- oder Hochbau?", 1931.

El objetivo de este artículo es entender hasta qué punto los conjuntos urbanos modernos fueron el resultado de una cultura urbana internacional y de la Carta de Atenas y cuál fue el papel que jugó el diseño urbano en la relativa pérdida de urbanidad apreciable tanto en el Este como en el Oeste cuando se produjo el crecimiento acelerado de las ciudades en los años 60 y 70 . Este texto se engloba en una investigación de carácter internacional desarrollada por sus autores como investigadores principales del proyecto I+D+i BIA2014-60059- $\mathrm{R}$ y en el marco en el marco del grupo PUPC de la Universidad de Zaragoza. Una visión más extensa se ofrecerá próximamente en un artículo en la revista Planning Perspectives y en un volumen coeditado por los autores de este artículo con el título Urban Visions: Cultures, Projects, Technologies, Landscapes (Prensas Universitarias de la Universidad de Zaragoza).

\section{Introducción}

En su libro El urbanismo en la Europa socialista (1971), el influyente arquitecto y teórico comunista Edmund Goldzamt afirmaba que el urbanismo en los países socialistas podía entenderse como una continuación de la tradición de las experiencias progresistas desarrolladas durante el siglo XX en Europa en el periodo de entreguerras. Goldzamt sostenía que "las ideas progresistas contenidas en (...) la Carta de Atenas pueden ser asumidas en unas condiciones de economía planificada socialista."1 Si consideramos aquellos innovadores episodios del urbanismo soviético o la arquitectura y el urbanismo de vanguardia que se desarrollaron en el periodo de entreguerras en Alemania, Hungría, Checoslovaquia o Polonia, su afirmación resulta convincente. De hecho, tras la Segunda Guerra Mundial, cuando se decidió dar respuesta a la creciente demanda de vivienda, aquellas ideas se aplicaron tanto en el urbanismo socialista (y por tanto en los conjuntos residenciales de las ciudades del Este) como en las ciudades occidentales. Por supuesto, existen diferencias obvias, pero también se pueden reconocer características comunes en las formas urbanas que se emplearon a ambos lados del telón de acero, sobre todo en los años 60 y 70, con la imposición generalizada de las visiones productivistas y "desarrollistas"2.

En cuanto a la terminología empleada para designar estos conjuntos residenciales, es necesario aclarar que estamos ante un concepto cuyo significado y definición varían dependiendo de cada país ${ }^{3}$. Por otro lado, en la historiografía urbanística es posible encontrar muchos análisis de ejemplos locales, especialmente monografías sobre ciudades y análisis históricos de conjuntos residenciales, pero muy pocas visiones comparadas ${ }^{4}$. Sin embargo, si descuidamos la dimensión internacional de este episodio en el periodo en el que el urbanismo moderno y la moderna cultura urbanística se iban imponiendo en toda Europa, corremos el peligro de no reconocer la verdadera especificidad de esas formas urbanas en cada ciudad. Por otra parte, si consideramos cómo los ideales y técnicas del urbanismo moderno comenzaron a cambiar inmediatamente después de su formulación, y cómo fueron aplicados en distintos países después de la Segunda Guerra Mundial, podremos identificar paralelismos y diferencias entre las formas urbanas "capitalistas" y "socialistas".

Resumen pág 55 | Bibliografía pág 59

Javier Monclús es arquitecto y Catedrático de Urbanismo en la Escuela de Ingeniería y Arquitectura de la Universidad de Zaragoza. Anteriormente ha trabajado en la Expo Zaragoza 2008 (2005-2009) y fue profesor de urbanismo en la UPC (1980-2005). Ha publicado más de un centenar de trabajos sobre Urbanismo e Historia urbana, entre ellos: "International Exhibitions and Urban Design Paradigms", en M. Amati, R. Freestone (eds), Exhibitions and the development of Modern Planning Culture (2014) y Culture, Urbanism and Planning (2006) (coed.).

Carmen Díez es arquitecto y profesora Titular de Composición Arquitectónica en la Escuela de Ingeniería y Arquitectura de la Universidad de Zaragoza. Entre sus trabajos sobre Urbanismo e Historia Urbana: "Marks of Identity. Semantics of Social Housing: Hof vs. Siedlung", in AA.VV. Social Housing (2010); "Zoom in/Zoom out. Viena, Milán: dos edificios, dos ciudades", en C. Sambricio (ed.) La construcción de la ciudad liberal (2004); y "Reflexiones sobre la reconstrucción italiana a partir de la experiencia del Tiburtino en Roma". Arquitectura 301 (1995)..

\section{Palabras clave}

Europa Post-1945, urbanismo moderno, Movimiento Moderno, conjuntos vivienda masiva, conjuntos residenciales socialistas, urbanismo soviético, formas urbanas, diseño urbano

1 El urbanismo en la Europa socialista, Barcelona: Gustavo Gili, 1980, p.172. GOLDZAMT, E. , Urbanistyka krajów socjalistycznych: problemy spoleczne. Warszawa: Arkady, 1971; Städtebau sozialistischer Länder. Soziale Probleme, Berlin, Verlag für Bauwesen, 1974; Stuttgart: Deutsche Verlags Anstalt, 1975; L'urbanistica dei paesi socialisti. Città, territorio e struttura sociale. Milano: Gabriele Mazzotta editore, 1977. En su libro Goldzamt desarrolla ideas sociales y socialistas que había ya abordado en anteriores publicaciones tales como William Morris and the Social Origins of Modern Architecture (Warsaw, 1967) o Social Aspects of Town Planning and Housing in Italy (Warsaw, 1968).

2 Una version reducida de las consideraciones desarrolladas en este artículo se presentó en forma de comunicación breve al $12^{\text {th }}$ International Conference on Urban History Cities in Europe, Cities in the World (European Association for Urban History, Lisbon, Portugal, 3-6 September, 2014). Otras comunicaciones presentadas en la misma sesión, titulada "Iron Curtain Cities? Urban Space in Cold War Europe" se centraron en las conexiones entre las ciudades "socialistas" y "capitalistas" en la Europa de la segunda posguerra. Entre ellas, la de LE NORMAND, B. "The contested place of self-building in Yugoslavia's socialist cities." 
3 El Oxford Dictionary define "housing estate" como "A residential area in which the houses have all been planned and built at the same time". Es en el caso de los Grands Ensembles franceses donde se puede encontrar una reflexión más profunda acerca del significado de este concepto: me désigne pas un mode d'edification, mais plutôt une forme et un payssage caractérisé para un regroupment de barres et de tours sur un espace soumis aux règles du zonage", VIEILLARD-BARON, $H_{\text {., "Sur l'origine des grands ensembles", en }}$ DUFAUX, F., FOURCAUT, A. (eds.), Le mond des grands ensembles, Paris: Créaphis, 2004. Desde la perspectiva del planeamiento urbano podemos referirnos a la definición que de los polígonos de vivienda ofrece F. de Terán: "Actuaciones unitarias, compuestas po conjuntos de bloques semejantes, realizados en poco tiempo, sobre piezas de suelo de operación), a través de una gestión única y un proyecto de conjunto", en DE TERÁN F. Historia del urbanismo en España, vol. 3, s. XIX y XX, Madrid: Cátedra, 1999, p. 228.

${ }^{4}$ Entre la literatura existente, se pueden mencionar algunos análisis parciales centrados en episodios concretos, entre ellos: AA.VV., 10 Stories of collective housing. Graphical analysis of inspiring masterpieces, Vitoria: $\mathrm{a}+\mathrm{t}$ architectural publishers, 2013; también existen algunos estudios generales sobre las ciudades soviéticas, con un enfoque socialista, como el mencionado de GOLDZAMT, E., op. cit., un libro importante que sorprendentemente no ha sido traducido al inglés, o el BATER, J. H., The Soviet City, London: Edward Arnold, 1980; otras publicaciones abordan el urbanismo de posguerra, como MUMFORD, E., The CIAM Discourse on Urbanism, 19281960, Cambridge: MIT Press 2000; otras se centran en los conjuntos residenciales en Francia, como DUFAUX, F. y FOURCAUT, A. (eds.), Le monde des grands ensembles, París: Créaphis 2004; también existen algunas visiones globales sobre los conjuntos residenciales del s. XX, desarrolladas en forma de gran narrativa, como HALL, $P$., Cities of Tomorrow: An Intellectual History of Urban Planning and Design in the Twentieth Century, Hoboken, New Jersey: Blackwell, 1988, especialmente el capitulo 7 "The City of Towers"; algunos otros afrontan estudios más sistemáticos sobre el urbanismo moderno del siglo XX, como WARD, S., Planning the Twentieth-Century City, Chichester, Hoboken, New Jersey: J. Wiley \& Sons, 2002, que aporta una perspectiva comparada internacional; otros llaman la atención sobre los distintos modos en los que la arquitectura y el urbanismo interactúan con las politicas del Estado del Bienestar, como SWENARTON, M., AVERMAETE, T. y VAN DEN HEUVEL, D. (eds.), Architecture and the Welfare State, New York: Routledge, 2014; finalmente, algunas publicaciones recientes con un enfoque similar al nuestro se centran en los problemas de estos conjuntos residenciales en diferentes países y ofrecen un diagnóstico interesante, como es el caso de URBAN, F., Tower and Slab. Histories of Global Mass Housing, London: Routledge, 2012.

5 Este artículo se enmarca en el proyecto I+D+i BIA2014-60059-R (2014-2017), que lleva por título "Nuevos retos para las ciudades españolas: el legado de los conjuntos de vivienda moderna y opciones de su regeneración urbana. Especifidad y semejanzas con modelos europeos", y forma parte de un programa Tempus, en el que participan siete universidades europeas $y$ cinco ucranianas, y se desarrolla entre 2012 y 2015 (http://sehud.com/)

${ }^{6}$ Nuestra intención fue escoger ejemplos representativos de distintas tradiciones culturales urbanas localizados en diferentes ciudades europeas de una cierta complejidad. Una aproximación similar puede encontrarse en el caso de las exposiciones internacionales, que en lugar de ser "episodios urbanos excepcionales" pueden entenderse, al mismo tiempo, como paradigmas de diseño urbano. Ver MONCLÚS, J., "International
La primera parte del artículo plantea cuestiones fundamentales sobre las distintas percepciones que estas experiencias suscitaron y sobre los cambios de paradigma que se produjeron respecto a los modelos de referencia, a la vez que explora las implicaciones prácticas de los principios modernos sobre esa compleja realidad. La segunda parte ofrece un intento de valoración de cómo se asumió la herencia del Movimiento Moderno a ambos lados del Telón de Acero, partiendo de algunos ejemplos de conjuntos construidos en distintas ciudades europeas de acuerdo con aquellas ideas progresistas y funcionalistas, aun siendo conscientes de que los procesos reales de construcción más bien se correspondieron con una especie de "vulgata" de los ideales modernos.

Nuestras investigaciones están basadas en la comparación de principios urbanísticos y formas urbanas en diferentes casos de estudio de ciudades europeas del Este y del Oeste (Sheffield, Ámsterdam, París, Berlín Oeste, Milán, Madrid, Barcelona, Zaragoza, Moscú, Berlín Este, Cracovia, Praga, Kiev) y parten de un trabajo más amplio que no podemos reproducir aquí5. Los casos de estudio seleccionados fueron originalmente trece, lo que nos ha permitido establecer ciertas comparaciones. Se trata de grandes conjuntos paradigmáticos (con un mínimo de 1.000 viviendas) que, si bien pueden entenderse como modelos excepcionales, es precisamente esta excepcionalidad la que los convierte en buenos observatorios para el urbanismo moderno. ${ }^{6}$ Otro motivo para esta elección fue elegir conjuntos que hubieran experimentado intervenciones de regeneración o sido objeto de estudios con vistas a intervenciones futuras. ${ }^{7}$ Con una metodología ecléctica -fuentes secundarias junto a la observación directa de formas urbanas- analizamos algunos conjuntos construidos durante el periodo socialista en ciudades del Este en la época de crecimiento urbano acelerado (años 60 y 70) así como en ciudades europeas occidentales, en la línea de nuestras investigaciones anteriores. ${ }^{8}$ Siendo conscientes de la imposibilidad de ofrecer una exposición detallada de cada uno de estos conjuntos en este texto, lo que aquí presentamos es una síntesis de algunos de los casos estudiados.

Teniendo todo esto presente y en paralelo a la lectura que Rem Koolhaas propone en la Bienal de Venecia de 2014, Absorbing Modernity, efectuamos ciertas consideraciones sobre cómo las diferentes culturas urbanas respondieron a la "llamada de la modernidad" en el pasado siglo. En este sentido, nos hemos planteado algunas cuestiones que, en relación a los distintos procesos urbanos que experimentan las ciudades del Oeste y del Este de Europa, podrían resumirse en la siguiente pregunta: ¿podemos decir que dichos procesos se corresponden simplemente con diferentes fases de un desarrollo común de modernización urbana europea? También consideramos otras cuestiones más específicas, como las formas en las que los ideales modernos fueron adaptándose a los diferentes sistemas políticos y socioeconómicos, explorando el salto que se produjo, tanto en las ciudades del Oeste como en las del Este, entre las expectativas iniciales y las cuestionables realizaciones de las "utopías urbanas modernas". La cuestión principal aquí es la de la contribución y la responsabilidad de la "cultura urbanística moderna", en su sentido más amplio e incluyendo la cultura del diseño urbano, con sus distintas versiones en el Oeste y en el Este, especialmente en referencia a los conjuntos residenciales de la década de los sesenta. Por lo tanto, no abordamos directamente otros argumentos importantes como la situación de mercado, cuestiones sociológicas o tecnológicas, legislación urbanística, influencia de políticas de suelo, etc., sino que el objetivo es entender hasta qué punto los conjuntos urbanos modernos fueron el resultado de una cultura urbana internacional y de la Carta de Atenas y cuál fue el papel que jugó el diseño urbano en la significante pérdida de urbanidad apreciable tanto en el Este como en el Oeste cuando se produjo el crecimiento acelerado de las ciudades en los años 60 y 70 .

\section{Ideales modernos, vivienda colectiva moderna}

\section{0-1950: la imposición de las nuevas concepciones en urbanismo y en la vivienda colectiva. Paradojas y contradicciones}

Desde la década de los años veinte, el urbanismo moderno desarrolló innovaciones radicales en la vivienda y en las formas urbanas. El espíritu de ese "nuevo urbanismo" ha sido objeto de una extensa literatura académica que enfatiza su relación con determinadas visiones urbanas, como el bien conocido modelo de la Ville Contemporaine de Le Corbusier (1922), una propuesta radical para la ciudad de la era industrial. Aunque en esos años coexisten distintas propuestas, como la Verticalstadt de L. Hilberseimer (1924), se puede decir que las diferencias no son sustanciales (la zonificación sucede en vertical). Otras propuestas posteriores en la escala de barrio se corresponden con ese espíritu del nuevo urbanismo, en el que las manzanas urbanas con calles corredor convencionales aparecen completamente "fuera de moda". 'Walter Gropius, en su contribución al III CIAM, muestra con precisión científica los problemas de los viejos modelos residenciales (como las Mietkasernen en Berlín) y defiende el bloque lineal en altura como alternativa. [1] Como contrapartida, el sistema arterial de vías rápidas aísla los edificios en altura, 
y los espacios verdes se convierten en los elementos dominantes de los trazados modernos. Realmente, ese importante cambio de paradigma relacionado con el prestigio del taylorismo y del fordismo fue el que, entre otros factores, implicó nuevas formas de ordenar los espacios urbanos $^{10}$

Bajo esta perspectiva focalizada en las formas urbanas, podemos asumir que, tras la Segunda Guerra Mundial, a pesar de las diferentes tradiciones culturales y situaciones políticas, los nuevos conjuntos residenciales fueron proyectados de acuerdo con -o siguiendo estrictamente- los postulados del Movimiento Moderno. Por supuesto este fue un proceso complejo, contradictorio y paradójico, ya que los grandes conjuntos residenciales se construyeron de acuerdo a los principios de los CIAM precisamente cuando dichos principios comenzaban a ser rechazados. En este sentido, si pudiéramos identificar una "hora cero" como punto de partida para la revisión más o menos radical de la vanguardia urbana, deberíamos situarla en los años 1950/51. En 1951 los 16 Principios del Urbanismo fueron aprobados en Berlín Este. ${ }^{11}$ Casi al mismo tiempo, en 1951, tuvo lugar el CIAM 8, The Heart of the City.

Es interesante advertir cómo la arquitectura en tiempos de Stalin, nada más terminar la Segunda Guerra Mundial, intentó establecer diferencias entre la visión soviética y el urbanismo capitalista del Oeste. En este contexto y como contrapunto a la Carta de Atenas, los mencionados principios combinaron las ideas del urbanismo moderno de los años treinta con las influencias que la era Stalin estaba dejando en los países del Este y quizá también con algunas de las ideas revisionistas presentadas en el CIAM 8 sobre la importancia de la dimensión cultural de las ciudades. ${ }^{12}$ Sin embargo, aquellos principios no estaban relacionados solamente con la necesidad de expresar valores socialistas, sino que también cuestionaban el urbanismo funcionalista de los CIAM. ${ }^{13}$

En conclusión, un primer cambio de paradigma se produjo entre 1933-43, pero la rigidez de los principios modernos condujo rápidamente a un segundo cambio tras la Segunda Guerra Mundial, apoyado por una "segunda generación" de arquitectos y urbanistas más sensible a los valores de las ciudades históricas, a sus centros y "corazones", al contraste entre la calidad de los espacios urbanos tradicionales y la desnudez de los modernos, a la falta de urbanidad en los nuevos conjuntos, etc. Aunque, como se verá a continuación, esto podría considerarse como un fenómeno transitorio de "vuelta al orden".

Por el contario, un análisis comparado más profundo de estos dos documentos confirma que algunos puntos en los Principios Socialistas -especialmente los relacionados con el tema de la vivienda- son bastante similares a los propuestos en la Carta de Atenas, como por ejemplo el urbanismo entendido como instrumento para organizar trabajo, vivienda, cultura y ocio (n. 2); el centro como corazón de la vida política, con edificios administrativos y culturales (n. 6); el tráfico como parte de la ciudad y en armonía con ella (n. 8); barrios con equipamientos comunes de tipo social, cultural y asistencial (n. 10); principios higiénicos (n. 11), etc. ${ }^{14}$

Todo ello nos lleva a afirmar que, a pesar de que la historiografía urbana ha generado una extensa literatura acerca de este proceso revisionista, ${ }^{15}$ el debate sobre el impacto de la Carta de Atenas en los conjuntos de vivienda construidos tras la Segunda Guerra Mundial permanece abierto. ${ }^{16}$

\section{0-1980: de la rápida adopción de los principios modernos a la de visiones críticas a partir de los años sesenta}

Revisando dos textos publicados a principios de los años sesenta en Berlín occidental y oriental, respectivamente, podemos focalizar nuestras reflexiones en la rapidez con la que las visiones utópicas se transformaron en voces críticas y negativas. En Berlín occidental, la publicación en 1964 del libro Die gemordete Stadt, de Wolf Jobst Siedler con fotografías de Elisabeth Niggemeyer, fue un hito importante. Un año antes, En la ciudad del mañana, basada en la correspondencia entre Brigitte Reimann y Hermann Henselmann, ya mostraba la aparición de otras visiones críticas en el lado este de la ciudad. Sin embargo, las críticas procedían de todas partes: los textos de Jane Jacobs (1961), Gordon Cullen (1961), Cristopher Alexander (1964), Alexander Mitscherlich (1965) o Aldo Rossi (1966) ilustran perfectamente la proliferación de dichas visiones y el rechazo de determinados aspectos del urbanismo moderno, especialmente de los grandes conjuntos de vivienda colectiva construidos en la década de los años sesenta, tanto en el Este como en el Oeste.

Respecto a esos conjuntos de vivienda, los problemas surgieron ya en la década de los setenta en las ciudades norteamericanas y europeas contemporáneamente, lo que dio lugar a un rechazo generalizado de los mismos. En realidad, las decisiones arquitectónicas y urbanísticas a menudo se interpretan confusamente y se las responsabiliza de problemas sociológicos de una forma superficial. Así ocurre en algunos textos de autores como Alice Coleman. ${ }^{17}$ En consecuencia,
Exhibitions and Urban Design Paradigms", en FREESTONE, R., AMATI, M. (eds.) Exhibitions and the Development of Modern Planning Culture, op. cit.

${ }^{7}$ Por ejemplo, el enfoque urbano, geográfico e histórico desarrollado por RONCAYOLO,

M. en "La ville aujourd'hui", en DUBY, G. (ed), Histoire de la France urbaine, 5, Paris: Seuil, 1985.

${ }^{8}$ MONCLÚS, J., LABARTA, C., DIEEZ, C., AGUSTín, L., BERGERA, I. (eds.), Paisajes urbanos residenciales en la Zaragoza contemporánea, Zaragoza: Prensas Universidad de Zaragoza, 2013; GUÀRDIA, M., MONCLÚS, J., OYÓN, J.L. (dir.), Atlas histórico de ciudades europeas, vol. I Península Ibérica, Barcelona: CCCB-Salvat, 1994; vol. II Francia, Barcelona-Paris: CCCBSalvat-Hachette, 1996; DiEZ, C. "Marks of Identity. Semantics of Social Housing: Ho vs. Siedlung", en Social Housing, Madrid: Ediciones asimétricas, 2010, pp. 26-30; DíEZ, C., "La influencia centroeuropea y la Ley Salmón (1927-36)", en C. SAMBRICIO (ed.), Un siglo de vivienda social (1903-2003) Vol. I, Madrid: Nerea, 2003, pp. 138-161; "El modelo vienés", p. 172-175; "Barrio de la Estrella", Vol. II, p. 114-115.

${ }^{9}$ HEBBERT, M., "The street as locus of collective memory", en Environment and Planning D: Society and Space 23 (4), 2005 p. 581-596.

10 HILPERT, T., La ciudad funcional. Le Corbusier y su visión de la ciudad, (1978) Madrid: IEAL, 1983; MONCLÚS, J. "International Exhibitions and Urban Design Paradigms", en FREESTONE, R., AMATI, M. (eds.), Exhibitions and the Development of Modern Planning Culture, op. cit.

11 "Grundsätze des Städtebaus. Von der Regierung der Deutschen Demokratischen Republik am 27. Juli 1950 beschlossen", en VON BEYME, K., et al., Neue Städte aus Ruinen: Deutscher Städtebau der Nachkriegszeit, Munich: Deutscher Taschenbuch Verlag, 1991, pp. 30-31. Una traducción al inglés de los 16 Principios puede encontrarse en CLELLAND, D., "From ideology to disenchantment", Architectural Design, 52, 11/12, 1982, p.p 41-45. Un resumen de los mismos se encuentra en STROBEL, R. W., "from 'cosmopolitan fantasies' to 'national traditions': socialist realism in East Berlin", en NASR, J., VOLAIT, M., Urbanism. Imported or Exported?, NYSE: Willey, Hoboken, 2003.

12 Este documento puede vincularse al "realismo socialista", y se publicó cuando nuevos principios arquitectónicos y urbanísticos se expusieron para apoyar "e intento de Stalin de aplastar la emergente Modernidad, suplantando su legado con una nueva estética que ensalzaba las virtudes de una sociedad socialista." STROBEL, R. W., "From 'cosmopolitan fantasies' to 'national traditions': socialist realism in East Berlin", op. cit. (traducción de los autores).

13 La estructura alternativa propuesta para clasificar los nuevos principios es buena prueba de ello. En lugar de las cuatro funciones de la ciudad propuestas en la Carta de Atenas, la versión socialista aborda, en primer lugar, la naturaleza histórica de la ciudad; a continuación, las formas urbanas de los conjuntos residenciales y las características deseables; en tercer lugar, el "ambiente urbano" de la ciudad (en oposición al "no urbano" de la ciudad jardín) $y$, en cuarto, la base de un diseño urbano específico frente a diagramas urbanos más abstractos. Algunas divergencias respecto a la Carta de Atenas son evidentes, por ejemplo la vinculación de estos grandes conjuntos con la vinculación de estos grandes conjuntos con de la ciudad jardin (n. 12); la defensa de algunos elementos de diseño urbano, tales como la calle o la plaza (n. 9, n. 16); finalmente, la insistencia en la presencia de elementos históricos que contribuyen a preservar la identidad nacional (n. 1, n. 5 , n. 14). 


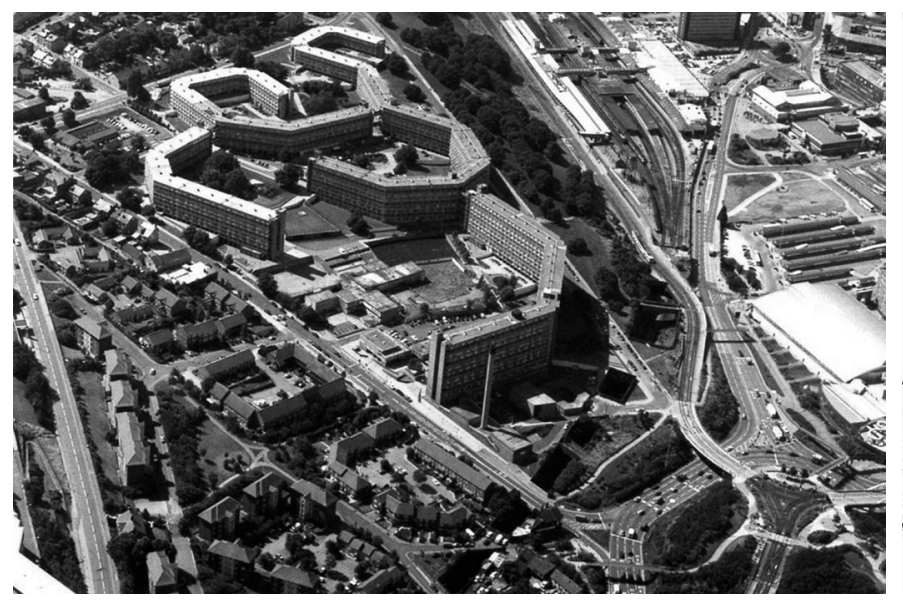

[2a]

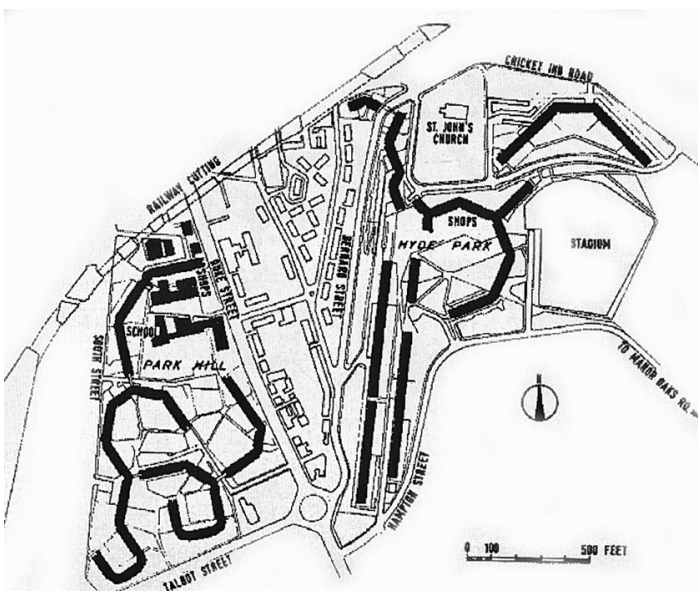

[2b] [2a-2b] Park Hill, Sheffield, 1954-1961. 1.000 viviendas en bloques de 4 a 13 plantas (24 ha). La radicalidad de los conceptos modernos, incluso en sus mejores ejemplos, condujo a una total estandarización de las soluciones estructurales, poniendo de manifiesto los limites de las apuestas megaestructurales. Fuente: Pérez Igualada, J., Manzanas, bloques $y$ casas. Formas construidas $y$ formas de suelo en la ciudad contemporánea, Valencia: UPV, 2005.

14 Autores como J. Rowell, R. W. Strobel o J. L. Sáinz Guerra son afines a esta posición que compartimos, que considera los 16 Principios del Urbanismo Socialista más como una derivación de la Carta de Atenas que como una propuesta alternativa. "La oposición radical a las ideas de la Carta de Atenas solo es parcial", en J. Rowell, "Les enjeux de l'importation d'une forme urbaine en RDA", DUFAUX, F., FOURCAUT, A. (eds.) Le monde des grands ensembles, op. cit., $p$. 105; SAINZ GUERRA, J. L., "Los cambios en la vivienda de la Ex-República Democrática Alemana a partir de la Reunificación", en Ciudades, 8, Valladolid: Instituto Universitario de Urbanística, 2004, pp. 63-85.

${ }^{15}$ MUMFORD, E., The CIAM Discourse on Urbanism, op. cit.; MUMFORD, E., "CIAM urbanism after the Athens charter", Planning Perspectives, Vol. 7, n. 4, 1992, pp. 391417; WAKEMAN, R., "Rethinking Postwar Planning History", Planning Perspectives, Vol. 29, n. 2, 2014, pp. 153-163; ORILLARD, C "The transnational building of urban design: interplay between genres of discourse in the Anglophone world", Planning Perspectives, Vol. 29, n. 2, 2014, pp. 209-229; ROGERS, E. N., "Le preesistenze ambientali e i temi pratici contemporanei", Casabella Continuità, n. 204, febbraio-marzo 1955, p. 3-6, etc.

16 PAQUOT, T. et al., "La Charte d'Athènes, et après?", Urbanisme, n. 330, op. cit.

17 COLEMAN, A., Utopia on trial: Vision and reality in planned housing, Londres: Hilary Shipman, 1985.

18 LAMPUGNANI, V. M., "Stadt oder Suburbia?", en ZARCH. Journal of Interdisciplinary Studies in Architecture and Urbanism, n. 5, Zaragoza: 2014, pp. 10-27.

19 “(...) high-rise housing should be seen as the result of a period in which planners and politicians aimed to build in the tradition of the CIAM movement..." en HELLEMAN, G., WASSENBERG, F., "The renewal of what was tomorrow's idealistic city. Ámsterdam's Bijlmermeer high-rise", Cities, vol. 21, 1, 2004 , pp. 3-17.

20 CAPEL, H., La morfología de las ciudades (vol. II), Aedes Facere: técnica, cultura y clase social en la construcción de edificios Barcelona: El Serbal, 2005, pp. 411-426.

21 LÓPEZ DE LUCIO, R., Vivienda colectiva, espacio público y ciudad. Evolución y crisis en el diseño de tejidos residenciales 1860 2010, Buenos Aires: Nobuco, 2013, p. 330. algunos episodios como la demolición del grupo Pruitt Igoe, en San Luis, se han interpretado como el canto de cisne del urbanismo moderno. A partir de ahí se han ido imponiendo las visiones críticas, como sucede en el importante libro de Peter Hall's Cities of Tomorrow (1988) y en otros muchos trabajos recientes, como el texto de Frank Wassenberg Large Housing Estates: Ideas, Rise, Fall and Recovery. También es interesante comprobar cómo, entre las visiones críticas contra el urbanismo de "torres y bloques", algunos arquitectos proponen la recuperación de la manzana incluso en las nuevas periferias urbanas (es el caso Vittorio Magnago Lampugnani en su último proyecto urbano para Zúrich). ${ }^{18}$

Con algunas variantes, la mayor parte de las visiones críticas coinciden al identificar los problemas y las debilidades de los conjuntos modernos. Como se puede comprobar, el paso de las grandes expectativas que había suscitado el urbanismo moderno a su cuestionamiento y posterior verificación final del fracaso fue realmente breve y concluyente. Tomando este largo e intenso debate como punto de partida, nuestra contribución ofrece una visión diacrónica y comparativa, focalizada en los problemas principales de ese tipo de conjuntos residenciales.

\section{Conjuntos residenciales modernos en Europa occidental: ¿experimentos CIAM, "errores de la cultura urbana moderna" o "pobreza de diseño"?}

La construcción y proliferación de grandes conjuntos de viviendas fue excepcional en toda Europa durante los años sesenta y setenta del siglo pasado. En primer lugar, debido a la escasez crítica de vivienda en un periodo de intenso crecimiento urbano y a la voluntad de resolver el problema con determinación. Por otro lado, tanto los urbanistas como los gobiernos se habían convencido de la conveniencia de las tesis de los CIAM $^{19}$, puesto que la estandarización y la prefabricación, así como los principios higienistas, permitían construir rápidamente. Pero el modelo moderno de vivienda colectiva trajo consigo serios problemas, culminando en la paradoja de que la mayor parte de los grandes conjuntos se construyeron de acuerdo con la Carta de Atenas precisamente cuando esos principios comenzaban a ser progresivamente rechazados, sobre todo durante la década de los años sesenta. ${ }^{20}$ Como señala R. López Lucio, la arquitectura y el urbanismo modernos produjeron la indiscutible mejora de la calidad de vida (sol, aire...), pero también dieron lugar a excesos de innovaciones urbanas radicales (rechazo de la calle, sobredimensionamiento de los espacios libres, etc.), ${ }^{21}$ aunque el problema fundamental radica en la rápida adopción de versiones simplificadas de estos principios en el contexto de un crecimiento urbano acelerado. ${ }^{22}$. En cualquier caso, como apunta Florian Urban, "alrededor de 1970 los bloques de vivienda proliferaron por todo el mundo (...), un signo visible del progreso y de la promesa de un mundo mejor en un futuro no tan lejano. Con su fuerza unificadora, estaban desafiando la dicotomía de la Guerra Fría."23

A pesar de la extensa literatura existente, todavía no están claros los motivos de los problemas y las debilidades de los conjuntos en ambos lados del Telón de Acero de Atenas cuarenta años más tarde de ser formulados, precisamente, en los años de la gran aceleración urbana y constructiva. ¿Se debieron fundamentalmente a la vulgarización de los principios de los CIAM, a los errores de la moderna cultura urbanística o, más bien, a la pobreza generalizada de un diseño urbano y de una arquitectura que confiaba en la potencia de la repetición y la estandarización? Intentando encontrar posibles respuestas a estas preguntas, hemos analizado algunos casos de estudio con el fin de entender hasta qué punto las estrategias y formas urbanas desarrolladas en estos conjuntos fueron diferentes -o no tanto- en las ciudades del Este y del Oeste.

Los casos de estudio que hemos analizado, de algunos de los cuales vamos a exponer aquí solo un 
[3a]
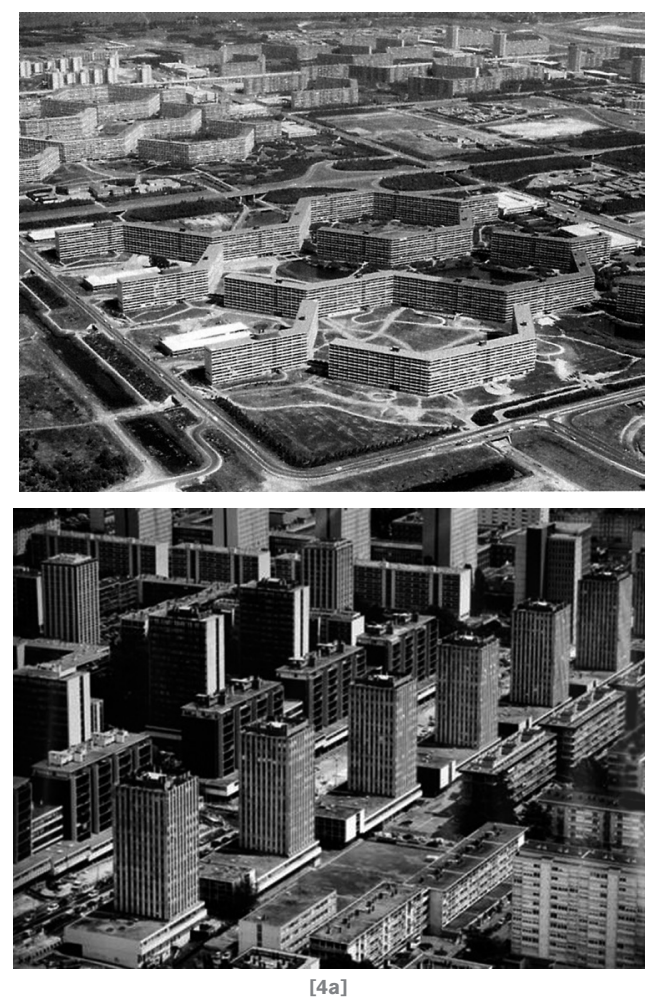

[3b]
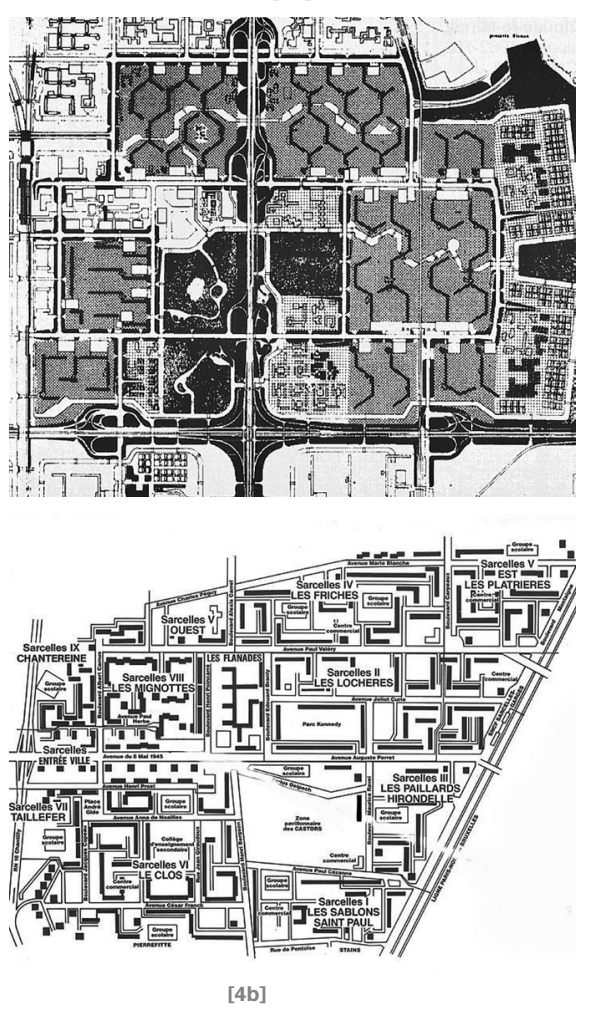

[4b]

breve comentario, muestran la distancia entre la expectación generada y los problemas a que su construcción dio lugar. Como en otros países occidentales europeos, en Reino Unido la tradición moderna estaba profundamente arraigada, y el Movimiento Moderno se convirtió finalmente en la ortodoxia de la década de los sesenta ${ }^{24}$, en la expresión del "sueño lecorbusieriano". ${ }^{25} \mathrm{La}$ cuestión es que "El Movimiento Moderno acabó por asociarse a la vivienda social y a la residencia de quienes no tenían elección". ${ }^{6}$ En Park Hill, Sheffield (1954-61), se pasó de una cualificada aprobación general a la decadencia, tras sufrir las consecuencias del declive de la industria del acero. También otros aspectos, como las políticas urbanas municipales aplicadas, contribuyeron a dañar el original ideal de igualdad, convirtiéndolo en el gueto de las clases marginales. ${ }^{27} \mathrm{Sin}$ embargo, su tamaño medio ha permitido posteriormente un control más asequible de los espacios públicos y ha facilitado el desarrollo de programas de regeneración ${ }^{28}$. [2]

Bijlmermeer, Amsterdam (1966), se presentó como solución de "nueva periferia avanzada en la edad de la máquina", todo un logro para el racionalismo más radical29; pero este icono moderno enseguida se convirtió en un error reconocido. ¿Qué es lo que falló? En este caso, la construcción coincidió con los grandes flujos migratorios producidos como consecuencia de la independencia de la antigua colonia holandesa de Surinam en 1975. Pero esta no puede ser la única explicación. Bijlmermeer demuestra cómo los proyectos se vuelven más complejos cuando la escala aumenta y los conceptos se radicalizan. ${ }^{30}$ Por supuesto, el urbanismo y la arquitectura no fueron los únicos responsables de estos problemas, pero pusieron de manifiesto los límites de estas megaestructuras. El proceso de regeneración urbana que se está llevando a cabo atiende fundamentalmente a la mejora de este aspecto. ${ }^{31}$ [3]

Francia merece especial atención debido al particular episodio de los Grands Ensembles. ${ }^{32}$ La abundante literatura existente pone de manifiesto las duras críticas a los conceptos de la modernización radical y a la excesiva estandarización de las soluciones arquitectónicas. ${ }^{33} \mathrm{~A}$ partir de 1958 se aprueban las ZUP ${ }^{34}$, siendo en este marco el caso más significativo Sarcelles (iniciado en 1958), un conjunto residencial con una composición monolítica en bloques horizontales organizados en una rígida retícula, en complicidad con los principios racionalistas de los CIAM. Las críticas a mediados de los años sesenta eran ya generalizadas. El hecho de que se haya inventado un término peyorativo para designar este fenómeno, la "sarcellitis"35, es un síntoma de que en el caso de París a la gran escala se suma otro factor decisivo: el gran número de conjuntos que generan paisajes banales en una parte considerable de las banlieues francesas. [4]

Menos atención han merecido generalmente las experiencias de conjuntos residenciales del Sur de Europa. En Italia, la tradición moderna se produce con notable continuidad tras la Segunda Guerra Mundial, convirtiéndose en la nueva ortodoxia para el urbanismo de los años sesenta. ${ }^{36}$ Con el desarrollo económico del "milagro italiano" se produjo, como en otras ciudades del sur de Europa, una explosión de las periferias. Quarto Cagnino en Milán (1967-73) constituye una

[3a-3b] Bijlmermeer, Amsterdam, 1966-72. 13.000 viviendas en bloques de 10 plantas $(650$ ha). "Nueva periferia avanzada en la edad de la máquina", todo un logro para el racionalismo más radical, que entró en crisis rápidamente en conjuntos gigantescos construidos de forma unitaria como gran megaestructura. Fuente: Pérez Igualada, J., 2005. Manzanas, bloques y casas. Formas construidas y formas de suelo en la ciudad contemporánea, Valencia: UPV, 2005.

[4a-4b] Sarcelles, París, 1955-70. 12.400 viviendas en bloques de 3 a 14 plantas. E episodio de los grands ensembles puede verse como uno de los productos más emblemáticos del urbanismo público francés. El gran número de conjuntos construidos es un reto adicional que se suma a la excesiva estandarización de las soluciones arquitectónicas y a la gran escala. Fuente: Wikimedia Commons.

[5a-5b] Quarto Cagnino, Milan, 1967-73. 1.100 viviendas en bloques de 8 plantas $(23,6$ ha). Un ejemplo poco común de programa coordinado como alternativa al modelo de barrio autosuficiente. Su localización en un apéndice marginal de la periferia milanesa dificulta su integración en la ciudad. Fuente: L'architettura cronache e storia n. 248, anno XXIII, giugno 1976 y Pugliese, R. (ed.), La casa popolare in Lombardia, Milano, 2005 Colección privada de los autores.

[6] Gran San Blas, Madrid, 1958-63. 10.442 viviendas en bloques de 3 a 6 plantas ( $84 \mathrm{ha}$ ). Este conjunto, el mayor construido en España en aquellos años, se basa en el concepto básco de distrito dividido en unidades vecinales o "parcelas". Cada una de ellas fue encargada a un equipo diferente de arquitectos, con distintas actitudes proyectuales frente a los principios de la Carta de Atenas. Problemas similares se produjeron independientemente de la unidad vecinal y, por tanto, de la solución urbanística. Fuente: fotografía de los autores 2014.

[7] Sudoeste del Besós, Barcelona, 1959-61. 8.234 unidades en bloques de 2 a 14 plantas $(40 \mathrm{ha})$. A pesar de algunos problemas iniciales en relación a la integración con la ciudad, más tarde la situación mejoró con el crecimiento y la consolidación del tejido urbano de la derecha del Ensanche barcelonés. Los problemas fueron mayores en lo que respecta a la calidad de los espacios intermedios, aunque como evidencia una reciente renovación, estos son susceptibles de importantes mejoras. Fuente: Ferrer, A., "The undeserved credit of the housing estate". Contemporary Barcelona 1856-1999. Barcelona: CCCB, 1996.

[8] Balsas de Ebro Viejo, Zaragoza, 1964-70. 1.534 viviendas en bloques de 4 plantas y torres de 12 (12,58 ha). La imagen global de 1970 muestra el conjunto tras ser inaugurado. Los edificios se conservan bien, pero la falta de algunas instalaciones, como los ascensores, ha desequilibrado la ocupación social del barrio. Muchas pequeñas tiendas que ayudaban a configurar los pequeños jardines $y$ plazas están hoy cerradas y abandonadas. Sin embargo, la coherencia global del conjunto permite mejors sustanciales mediante interpenciones puntules de regeneraco venciones puntuales de regeneración urbana, respetando el plan original. Fuente: Colección privada de los autores.

[9a-9b] Bienczyce, Nowa Huta, Cracow, 1958-62. 12.500 unidades. Los conceptos del urbanismo soviético de los años veinte y

22 Un interesante análisis sobre las debilidades y oportunidades en las Großsiedlungen se puede encontrar en los estudios sistemáticos sobre la Gropiusstadt en Berlín, proyecto de W. Gropius y su equipo TAC. Ver BIELKA, F., BECK, C. (eds.) Heimat Großsiedlung. 50 Jahre Gropiusstadt Entwicklungsmöglichkeiten für die Zukunft, Berlin: Nikolai Verlag, 2012.

${ }^{23}$ URBAN, F., Tower and Slab, op. cit., p.17. (traducción de los autores). 
[5a]

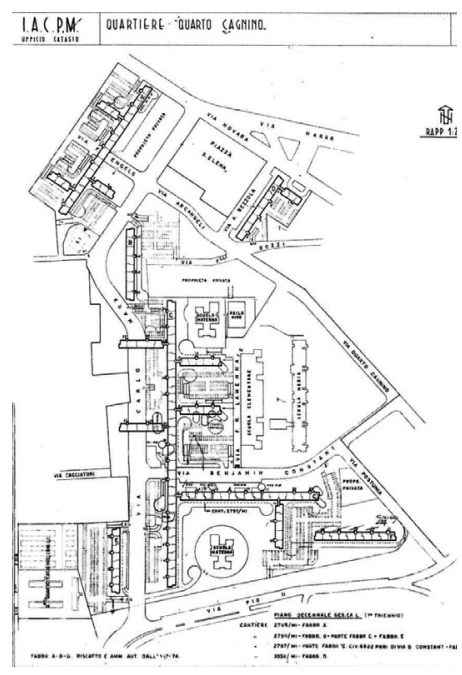

[5b]
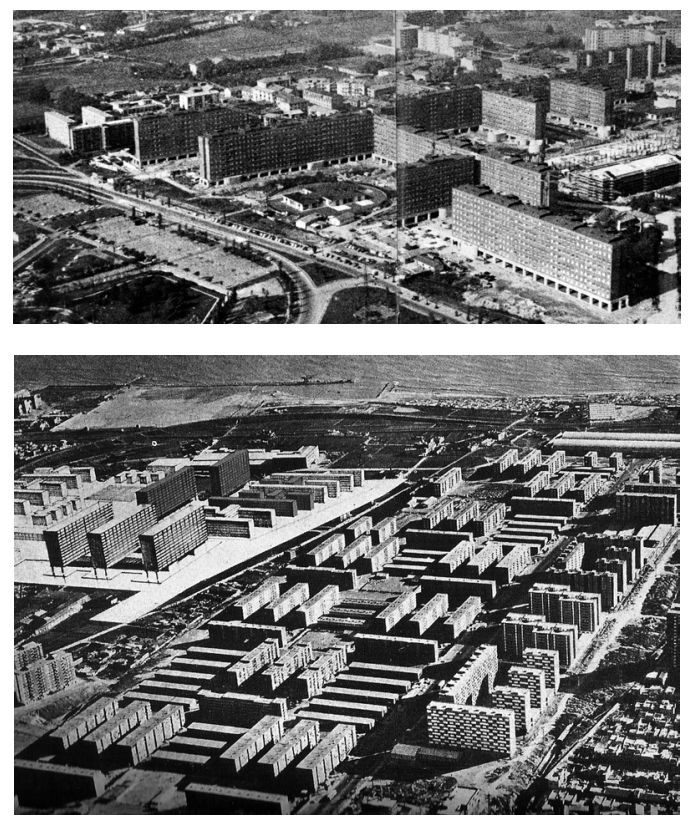

[7]
[6]
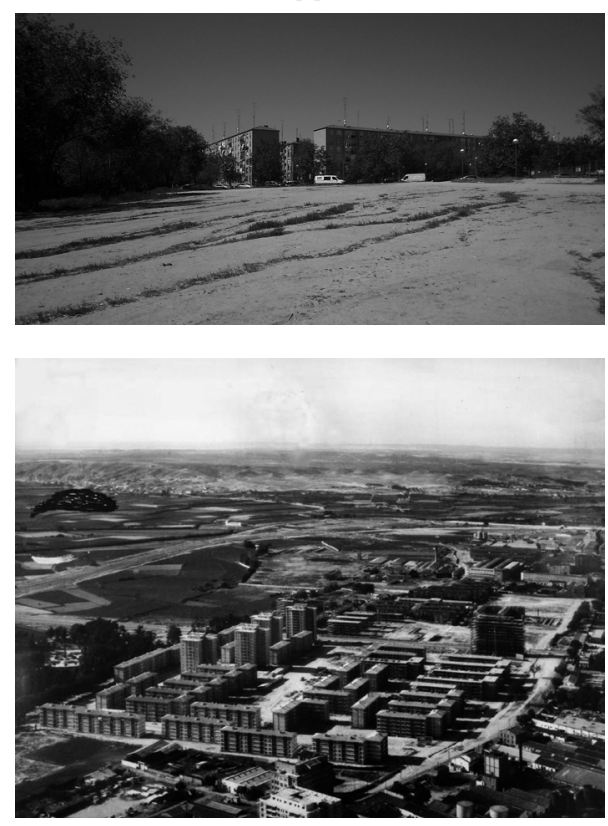

[8]
24 WARD, S., Planning the TwentiethCentury City, Chichester, UK: J. Wiley \& Sons, 2002, p. 241. Conceptos como "unidad de habitación", jerarquia, recintos autónomos, espacios abiertos y modernos conjuntos urbanos residenciales se incluyeron en el prestigioso County of London Plan de 1943.

$\mathbf{2 5}$ HALL, P., Cities of Tomorrow: An Intellectual History of Urban Planning and Design in the Twentieth Century, Hoboken, New Jersey: Blackwell, 1990. Nos referimos a conjuntos como el de Roehampton en el Oeste de Londres -Alton East and Alton West, 1953-y a otros episodios como el concurso para nuevas viviendas en Golden Lane, Londres (1952) o el de Robin Hood Gardens (1966-72), ambos desarrollados por los Smithson, así como a algunos otros proyectos vinculados al trabajo del Team $X$ ampliamente estudiados por historiadores $y$ arquitectos.

${ }^{26}$ GOLD, J. R., The Experience of Modernism: Modern architects and urban transformation, 1954-1972, New York: Routledge, 2007, p. 170. (traducción de los autores).

27 BLUNDELL JONES, P., "Second chance for Sheffield's streets in the sky", Architectural Review, 21.09.2011.

28 Acerca de las intervenciones de regeneración que se están llevando a cabo, ver Urbansplash transformation, Riba Publishing http://www.urbansplash.co.uk/ residential/park-hill, último acceso diciembre 2014

${ }^{29}$ WAGENAAR, C., Town Planning in the Netherlands since 1800: Response to Enlightenment Ideas and Geopolitical Realities, Rotterdam: 010 Publishers, 2011 p. 430; IBELINGS, H., 20th Century Urban Design in the Netherlands, Rotterdam: NAI, 1999

${ }^{30}$ Enormes espacios abiertos, segregación del tráfico (en tres niveles), un número alto de unidades de habitación (13.000) con variaciones tipológicas mínimas, megaestructuras de corredores, severas geometrias, etc. Como afirma Rem Koohaas: "with its segregation of traffic, its elevated highway, metro, green grounds, rational apartments, the Bijlmer represents a particular architectural doctrine -codified most memorably by CIAM in the thirties- realized in retrospect... So near and yet so far... Prewar CIAM urbanism realized in the late sixties", KOOLHAAS, R., S, M, L, XL, New York: The Monacelli Press, 1995, p. 863.

${ }^{31}$ Bijlmermeer Renovation Planning Office (Projectbureau Vernieuwing Bijlmermeer), The Bijlmermeer Renovation, Facts \& figures alternativa al modelo de barrio autosuficiente. ${ }^{37}$ Este conjunto, como ocurrió en otros de los casos de estudio mencionados, tuvo que enfrentarse a problemas de distinta índole, jurídicos o económicos, derivados no necesariamente de los aspectos urbanísticos o arquitectónicos. La venta del barrio a una empresa semi-privada ha contribuido a mejorar tanto los edificios como los espacios públicos, al quedar el mantenimiento en manos del condominio. ${ }^{38}$ A pesar de ello, los condicionantes iniciales son tan fuertes que ponen de manifiesto los límites de las intervenciones realizadas. [5]

Las ciudades españolas constituyen laboratorios interesantes para estudiar estas formas urbanas y no difieren demasiado de los ejemplos mencionados anteriormente. Como señalaba en un afinado texto R. Moneo: "En esa estructura, como valioso escalón intermedio entre la ciudadmunicipio y la familia-casa, se introduce ahora el barrio-polígono: la "neighborhood unit" de los urbanistas ingleses, las "Siedlungen" de los alemanes o los "grands-ensembles" de los franceses quedaban así bautizados, si bien con la ambigüedad que suponía el calificarlos simplemente, recurriendo tan solo al auxilio de la geometría, como polígonos." ${ }^{39}$

El Gran San Blas (1958-1963) ${ }^{40}$, al Este de Madrid se basa en el concepto básico de distrito dividido en unidades vecinales o "parcelas". Cada una de ellas fue encargada a un equipo diferente de arquitectos, todos trabajando bajo las directrices del Plan Parcial. Los diferentes proyectos ponen de manifiesto que no hubo una aproximación conjunta, y esto nos permite reconocer distintas actitudes frente a los principios de la Carta de Atenas. Resulta interesante verificar que el barrio presenta en su conjunto problemas que se producen independientemente de la unidad vecinal de que se trate y, por tanto, de la solución urbanística. Los problemas son similares también a otros ejemplos mencionados antes. Algunos programas de renovación han mejorado las deficiencias de los edificios, pero los espacios públicos permanecen en parte infrautilizados o abandonados. [6]

El polígono de San Martí del Besós (1958-1961) en Barcelona puede servir como ejemplo de polígono prototípico construido en la década de los sesenta. ${ }^{42}$ En los círculos profesionales fue considerado, junto con Montbau, relativamente ejemplar (aunque con una calidad arquitectónica menor que este último). En la descripción del polígono promovido por el Patronato Municipal de la Vivienda se hace referencia a un "un trazado urbanístico a base de grandes bloques concebidos como unidades de habitación." ${ }^{43}$ ¿Qué falló en este caso? Como ocurrió en Quarto Cagnino, hubo algunos problemas al principio respecto a la integración con la ciudad, aunque más tarde la situación mejoró con el crecimiento y la consolidación del tejido urbano. Los problemas fueron mayores en lo que respecta a la calidad de los espacios intermedios, pero como evidencia una reciente renovación, estos no son difíciles de mejorar. [7]

Al comienzo de los años sesenta, el crecimiento urbano de Zaragoza fue canalizado a través de actuaciones aisladas o de planes de desarrollo del Instituto Nacional de la Vivienda. Balsas de Ebro Viejo (1964-1970)44, el último gran conjunto construido en Zaragoza, está basado en una propuesta claramente moderna y marca un salto cualitativo comparado con otros proyectos previos. ${ }^{45}$ ¿Cuál fue el problema en este caso? Como en los casos anteriores, aunque los espacios 


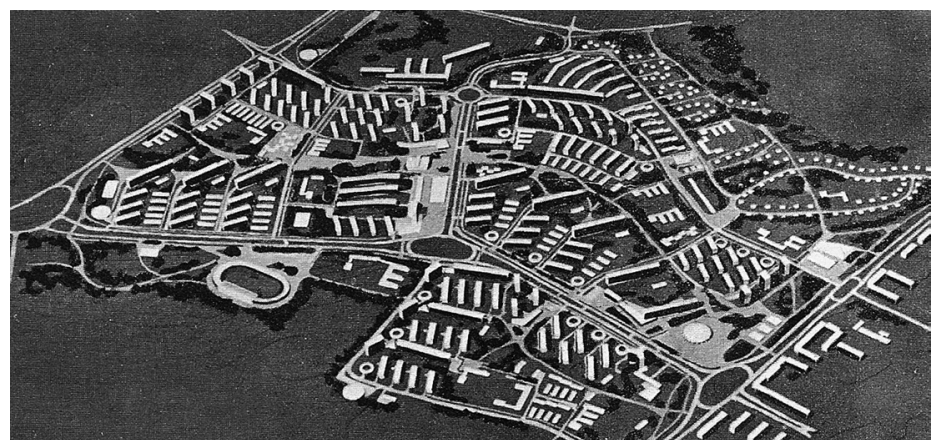

[9a]

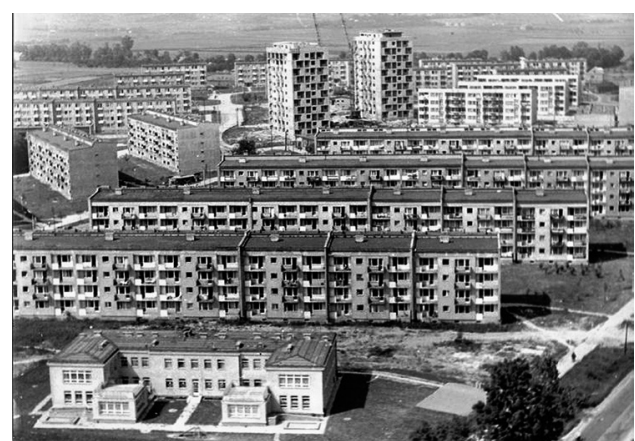

[9b] urbanos mantienen su carácter doméstico, al ser "tierra de nadie", carecen de mantenimiento y cualificación. Muchas pequeñas tiendas que ayudaban a configurar los pequeños jardines y plazas están hoy cerradas y abandonadas. Los edificios se conservan bien, pero la falta de algunas instalaciones como los ascensores ha desequilibrado la ocupación social del barrio. Sin embargo, la coherencia global del conjunto permite mejoras sustanciales mediante intervenciones puntuales de regeneración urbana, respetando el plan original. [8]

El análisis de estos ejemplos españoles vuelve a mostrar la heterogeneidad de las estrategias proyectuales y de las formas urbanas resultantes. Aunque generalmente se conciben como fragmentos relativamente autónomos, consecuentemente con su carácter de piezas proyectadas y gestionadas de manera unitaria, algunos se integran mejor que otros en su entorno. Ciertamente, casi todos ellos poseen un carácter bastante monolítico asociado a una escasa oferta tipológica, pero existe gran diversidad en las composiciones de los conjuntos, en la disposición y escala de los espacios intermedios, en la adaptación a las condiciones topográficas o en la propia arquitectura de los bloques y torres que contribuye a su definición morfológica. En cualquier caso, es importante diferenciar esos ejemplos, bastante notables, de la "avalancha de proyectos arquitectónicos de baja calidad" que caracterizan la mayor parte de las nuevas periferias residenciales de las ciudades españolas. ${ }^{46}$

\section{Conjuntos residenciales modernos y urbanismo del Este}

Al abordar la cuestión del urbanismo residencial socialista, es necesario considerar la situación de la Europa del Este después de la Segunda Guerra Mundial. Como ocurría en el Oeste, fue en aquel momento cuando la escasez de viviendas era tan crítica que desencadenó un acelerado proceso de urbanización generalizado, aunque con algunas diferencias según las ciudades. Estas necesitaban crecer rápidamente, y los ideales del Movimiento Moderno (los principios de los CIAM) parecían responder perfectamente a las nuevas circunstancias.

Dos tipos de aproximación nos han servido para establecer paralelismos y diferencias entre las soluciones occidentales y las propuestas socialistas. En primer lugar, una revisión de la cultura urbanística en los países socialistas durante aquellos años, ya que algunas publicaciones importantes de sus protagonistas, como el mencionado y exhaustivo trabajo de Edmund Goldzamt, aportan pistas interesantes. En segundo lugar, la observación in situ de algunos de estos conjuntos, junto con visiones comparadas y documentos y literatura especializada, nos permiten establecer importantes paralelismos y matizar algunas diferencias formales que van más allá de la significación y de la dimensión global de este fenómeno.

Centrándonos en la estructura urbana, es importante señalar que el planeamiento urbanístico de los nuevos conjuntos empleó el concepto de "unidad vecinal" como base, al igual que ocurrió en el Oeste. El Plan de Moscú de 1935, que compartía completamente el espíritu de la Carta de Atenas, había ya establecido estos principios urbanos. ${ }^{47}$ Más tarde, en la planificación urbana soviética de la posguerra, el mikrorayon emergió como versión socialista de la unidad vecinal. ${ }^{48}$ Este modelo se ha consolidado de forma sistemática, aunque con algunas variantes, en forma de distritos y microdistritos, en consonancia con el concepto de comunidad ideal y autosuficiente considerada como la unidad básica del desarrollo residencial. Aparte de esta cuestión, podemos identificar otras importantes semejanzas con el urbanismo funcional y orgánico que llegó a dominar la escena internacional. ${ }^{49}$

En cualquier caso, si se focaliza el análisis en las formas urbanas, parece claro que los conjuntos residenciales en las ciudades del bloque socialista resultan ser expresión más bien del urbanismo moderno que de las políticas urbanas socialistas. $Y$ ello tiene que ver con el hecho de que las formulaciones doctrinales que están en la base de los conjuntos residenciales masivos son previas al establecimiento del socialismo en Europa y diferentes del urbanismo revolucionario soviético,
(2008). http://www.vernieuwdebijlmer.nl/ bijlmer11/index.php/component/docman/doc_ download/86-the-renewal-of-the-bijlmermeerfacts-a-figures, último acceso diciembre2014

32 En Francia, también un pais con indiscutible tradición moderna, este episodio que comenzó a tomar forma en los años cincuenta constituye el compromiso más radical y definitivo con la experiencia de los conjuntos residenciales y uno de los productos más emblemáticos del urbanismo oficial durante los "Trente Glorieuses" (19451975)

${ }^{33}$ En parte debido al uso prevalente de la prefabricación, lo que llevó a un rechazo generalizado en los años sesenta. DUFAUX, F., FOURCAUT, A., Le monde des grands ensembles, op. cit. Algunos números de la revista Urbanisme han tratado especificamente este tema: "Le grand ensemble, histoire et devenir", Urbanisme 322, 2002; "La Charte d'Athènes et après?", Urbanisme 332, 2003 ; "Paris/Banlieues", Urbanisme 333, 2003.

${ }^{34}$ Zonas a urbanizar con prioridad, que fijaron el perimetro de actuación mediante decreto ministerial. Esto condujo a un aumento considerable de la construcción: en 1964 existian ya 200 grandes conjuntos de más de 1.000 viviendas (95 en Paris). La introducción de criterios modernos de renovación urbana, en paralelo a las nuevas formas urbanas que se impusieron en la periferia residencial (banlieues), convierte a la capital de Francia en un caso de excepcional interés.

35 MERLIN, P., Les villes nouvelles en France, Paris: Puf, 1991, p. 330; 1945-1975, Une histoire de l'habitat. 40 ensembles "Patrimoine du XX siècle ", Exposition présentée à La Maison de l'architecture en Îlede-France, du 5 juillet au 15 septembre 2011; THOMÉ, P., (coord.), Créateurs d'utopies. Sarcelles. Les 50 ans du Grand Ensemble, Paris : Yves Michel, 2006; STÉBÉ, J.M. "Les grands ensembles: des cités radieuses aux zones urbaines sensibles", Universität Stuttgart, 2011.

http://www.uni-stuttgart.de/lingrom/archiv/ gallerie_archiv_veranstaltungen_pdf/ conference_stebe_10_11.pdf, último acceso diciembre 2014

${ }^{36}$ Con énfasis más en los "proyectos" que en los "planes". Ver BENEVOLO, L., GIURA, T., LONGO, MELOGRANI, C., I modelli di progettazione della città moderna. Tre lezioni, Venezia: Cluva, 1969

37 Un ejemplo inusual de programa

coordinado, financiado por aproximadamente 50 cooperativas y varios grupos, que persigue una solución orgánica y unitaria y un incremento de la calidad y accesibilidad de los servicios públicos. Ver MONTALDO, V., "Quartiere Gescal di Quarto Cagnino", en L'Architettura. Cronache e Storia 248, anno XXIII, giugno 1976, Roma: Mancosu, p. 80-85.

38 LUCCHINI, M., "Quartiere Quarto Cagnino", en La casa popolare, Milano: Ordine degli architetti, 2013, p. 266-267.

39 MONEO, R., Vivienda y Urbanismo en España, Madrid: Banco Hipotecario, 1982

${ }^{40}$ Es el mayor poligono residencial construido 
treinta se reconocen también en los conjuntos polacos. La gran escala de este conjunto y la organización autónoma de cada unidad residencial -osiedle-permiten que distintas residencia -osidle-permiten que dsti las unidades construidas en los cincuenta junto al urbanismo abierto de las unidades posteriores. Fuente: Goldzamt, E., El urbanismo en la Europa socialista, Barcelona: Gustavo Gili, 1980 y Wikimedia Commons.

[10] Invalidovna, Praga, 1950-65. 1.000 viviendas en bloques de 5 a 11 plantas (14

en España en ese periodo. (10.442 viviendas en bloques de 3 a 6 plantas en una superficie de 84 ha). Ver Gran San Blas. Análisis sociourbanístico de un barrio nuevo español, suplemento de Arquitectura 113-114, Madrid: COAM, mayo-junio 1968; LÓPEZ DE LUCIO R., "El Gran San Blas", en SAMBRICIO, C. (ed.), Un siglo de vivienda social, op. cit., vol 2; SAMBRICIO, C., LAMPREAVE, R. (eds.), 100 años de historia de la intervención pública en la vivienda y la ciudad, Madrid: AVS, 2008, p.136.

41 Construido en el Noreste de Barcelona (o Sudoeste del Besós, como fue denominado inicialmente), es el mayor polígono de viviendas Barcelona (8.234 viviendas en 84 ha). Pertenece a la categoría de "Polígonos de extensión residencial" construidos en la segunda mitad de los cincuenta: BUSQUETS, J., La construcción urbanística de una ciudad compacta, Barcelona: Serbal, 2004, p. 264

42 TENA, P., Universalidad y adecuación en la obra de LIGS, Tesis Doctoral Universidad Politécnica de Cataluña, 2010. http://www. tdx.cat/bitstream/handle/10803/6821/ TPTG1de3.pdf?sequence=1, último acceso diciembre de 2014

43 Cuadernos de Arquitectura y Urbanismo, 37. Barcelona: COAB, 1959. A pesar de ello, se criticó mucho, muy tempranamente y de forma explícita, la aplicación de los principios de la Carta de Atenas. Entre otras cuestiones, se atacó con dureza la "falta de coherencia", como acabamos de ver que sucedió en

Madrid. Las críticas fueron similares a las que surgieron en otras ciudades $y$, en este caso, incluian, sorprendentemente, el rechazo a densidades demasiado bajas (en comparación con las del Ensanche).

441.534 viviendas en bloques y torres de 4 a 12 plantas en una superficie de 40 ha. $S$ dimensión permitió ofrecer más viviendas y una mayor variedad de tipos residenciales. Ver Ramos, M., "Polígonos de vivienda", en MARCO, R.Y BUIL, C. (eds.), Zaragoza 1908-2008, Zaragoza: Institución Fernando el Católico, 2009, p. 258.

45 Incluye un grupo de torres de vivienda de 12 plantas. Con la clara intención de crear un nuevo perfil urbano, refuerza la imagen del conjunto Los principios de diseño urbano aparecen descritos en la memoria del proyecto: distancias entre bloques, disposición, altura y orientación de los bloques en función de optimizar las condiciones de luz y soleamiento, etc. DIEZ, C., PÉREZ

L "Balsas de Ebro Viejo, 1964-70",

MONCLÚS, J. (ed.), Paisajes residenciales urbanos, op. cit., pp. 61-63.

46 FERRER, A., "The undeserved credit of the housing estate", en Contemporary Barcelona 1856-1999, Barcelona: CСCB, 1996.

47 Incluso podría interpretarse como un desarrollo posterior de la Carta de Atenas. En los diez "Principios para planificar la ciudad socialista" el número 3 (Desarrollo planificado de las áreas residenciales) especifica las caracteristicas de las unidades residenciales. BATER, J. H., The Soviet City, Londres: Edward Arnold, 1980, pp. 27-28 (1. Limited city size, 2. State control of housing, 3. Planned development of residential areas, 4. Spatial equity in the distribution of items of collective consumption, 5 . Limited journey to work. 6. Stringent land-use zoning, 7. Rationalized traffic flow, 8. Extensive green space, 9. Symbolism and the central city, 10. Town planning as an integral part of national planning.)

${ }^{48}$ Estas unidades o microrayon (de 8.000

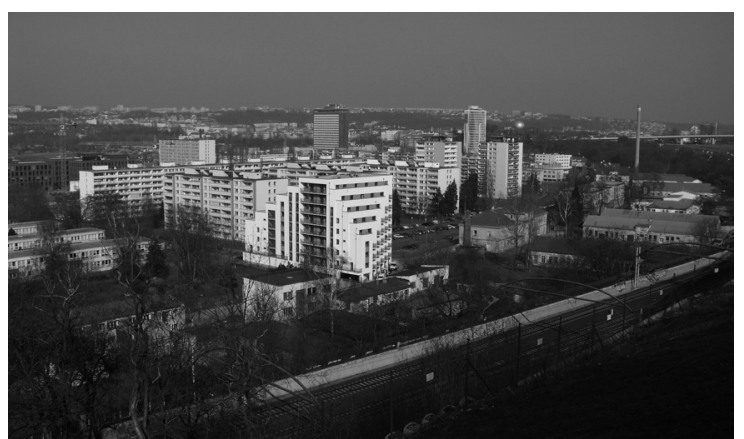

ha). A pesar del hecho de que el urbanismo monolítico de los Panelak fue dominante, cierto tipo de experimentación se desarrolló en los años centrales det periodo en los años centrales del periodo socialista dando lugar a algunas propuestas equivalentes a aquellas de mayor calidad en la cultura arquitectónica y urbanística internacional. Fuente: Wikimedia internaciona. $P$ Commons. [11a-11b] Rusanivska, Kiev, 1961-74. 11.250 unidades en bloques de 9 a 16 plantas ( 84 ha).

[10]

incluso si los conjuntos más grandes adoptaron el modelo de torres y bloques en todas partes. ${ }^{\mathbf{5 0}}$

Aquí la cuestión es que algunos cambios importantes tuvieron lugar después de 1955, cuando el urbanismo socialista adoptó incuestionablemente el "urbanismo abierto" y los modelos urbanos internacionales, y que aún no disponemos de un panorama completo del impacto que causó la cultura del urbanismo internacional en las ciudades del Este. ${ }^{51} \mathrm{~A}$ pesar de las importantes diferencias que se encuentran en el modo en que dichos principios se adoptaron en el Este, y del pragmatismo que caracterizó la producción de vivienda masiva, encontramos un gran nivel de experimentación, sobre todo en los años sesenta. Por otro lado, frente a la experimentación de entreguerras y, como ocurrió también en los países del Oeste de Europa, la producción de grandes conjuntos de vivienda se consolidó como una opción más pragmática que el "modelo de la ciudad socialista". Como apunta Stephen Ward, "...al final de los años cincuenta, la política de vivienda de la Alemania del Este dio un giro, cada vez más práctico, hacia la producción masiva. En 1960, los principios radicales de la modernidad y las conexiones de los años treinta con la Unión Soviética fueron redescubiertas. Siguiendo la línea soviética, las referencias a modelos urbanísticos occidentales se hicieron cada vez más comunes: ${ }^{12}$ De hecho, desde la década de los sesenta, los paisajes urbanos, y especialmente los suburbanos de las ciudades socialistas se han ido configurando mediante la proliferación de formas caracterizadas por la repetición de construcciones estándar. ${ }^{53}$ Como ocurre en las ciudades occidentales, pero con una proporción de la población muy superior viviendo en esos conjuntos en las ciudades socialistas (más de un $50 \%$ ), los grandes conjuntos residenciales construidos en el Este resultan ser "más duraderos y deficientes."54 Efectivamente, durante los años setenta y al principio de los ochenta, cuando la prefabricación aún dominaba, estos conjuntos continuaron construyéndose, aunque con más variedad y con diferentes escalas.

Lógicamente, es en Moscú donde se puede observar más continuidad con los principios de los CIAM, a pesar de los intentos de diferenciarse del Oeste durante el periodo estalinista. En la época de Kruschev, el compromiso con la industrialización y la prefabricación dio lugar a consecuencias bien conocidas en las formas urbanas de los conjuntos..$^{55}$ Los grandes desarrollos de viviendas se fragmentaron en unidades vecinales básicas (mikrorayons), que se organizaban de acuerdo a un esquema estructural común: espacios verdes internos con un modelo de construcción mixta, jugando con el contraste entre volúmenes bajos y torres verticales como una "solución" al problema de la monotonía. Los barrios Khimki-Khovrino, Fili-Izmajlovo o ChorosevoMnevniki, situados al noreste de Moscú y construidos en aquellos años, pueden mencionarse como conjuntos residenciales paradigmáticos de esas tendencias. Algo parecido ocurre en los conjuntos polacos. [9]

Praga es otra ciudad importante en la que la tradición moderna continuó antes y después de la Segunda Guerra Mundial. Conjuntos residenciales (sidliste) de las primeras generaciones como Solidarita (1947-49) o Invalidovna (1950-1965) son buena prueba de dicha continuidad. ${ }^{56}$ [10] A pesar del hecho de que el urbanismo monolítico de los Panelak fue dominante, cierto tipo de experimentación se desarrolló en los años centrales del periodo socialista, dando lugar a algunas propuestas equivalentes a aquellas de mayor calidad en la cultura internacional urbana y arquitectónica. El proyecto para Etarea es un episodio muy significativo a este respecto. ${ }^{57}$ Algunos conjuntos de gran escala más recientes como Bohnice ${ }^{58}$ o Jihozápadní Město vuelven a confirmar que estos conjuntos residenciales masivos responden a estándares funcionalistas y racionalistas, con bloques y torres que se alzan en grupos aislados en el interior de tranquilas zonas verdes equipadas con zonas de juego y jardines.

Para terminar, con el fin de comparar algunos procesos experimentados en Ucrania y Rusia, podemos tomar en consideración algunos conjuntos de Kiev y Lviv. Como ocurre en otros países socialistas, los grandes conjuntos residenciales continuaron siendo una importante parte del 


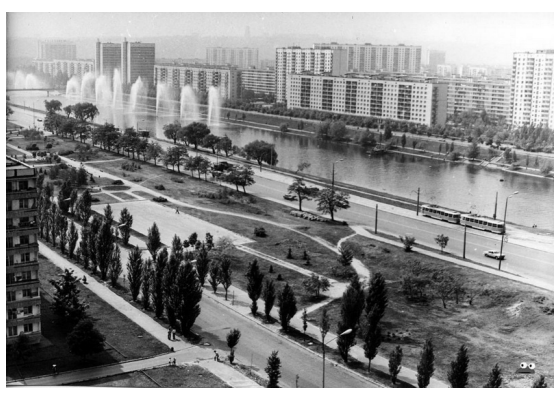

[11a]

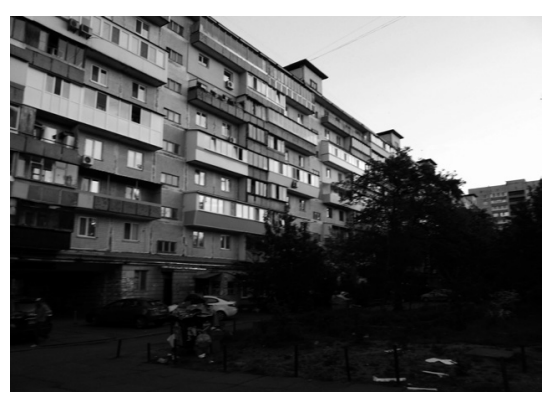

[11b]

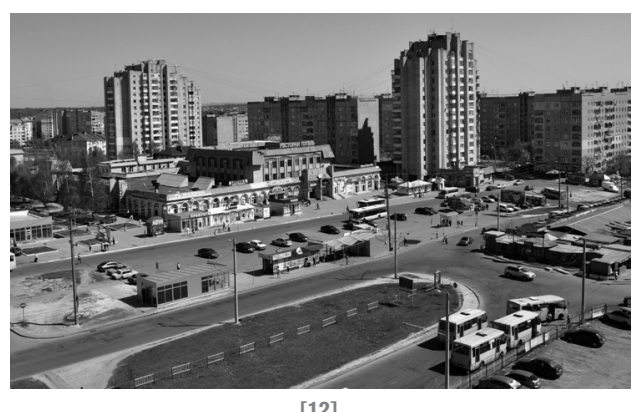

[12] stock total de viviendas, en una primera generación todavía influenciada por el Máster Plan de Moscú de 1935. ${ }^{59}$ Una experiencia singular digna de mención es el mikrorayon Rusanovska de Kiev (1961-74), proyectado como una ciudad dormitorio modelo no directamente vinculada a la industria. ${ }^{60}$ Construida en su mayor parte con bloques de entre 9 y 16 plantas, de paneles de hormigón, su mayor problema en la actualidad no son tanto los espacios públicos o el diseño urbano, cuanto la mala calidad de la construcción. [11] El barrio de Sykhivs'kyi, en Lviv, puede servirnos de comparación, como ejemplo de cómo los grandes espacios públicos son a menudo un problema, especialmente cuando la construcción de los equipamientos se retrasa. ${ }^{61}$ [12]

Para cerrar estos breves comentarios sobre los conjuntos residenciales construidos a ambo lados del Telón de acero, diremos que hemos desarrollado un análisis más directo y comparativo de aquellos conjuntos que hemos visitado y que conocemos mejor. Las ilustraciones que aquí se publican dan una idea de las morfologías urbanas y de las formas de los edificios residenciales en cuatro conjuntos paradigmáticos: Gran San Blas (Madrid), Ebro Viejo (Zaragoza), Sudoeste del Besós (Barcelona), Rusanivska (Kiev), Invalidovna (Praga).

\section{Similitudes y contrastes de un legado común}

Volviendo a la cuestión original acerca de las posibles similitudes y contrastes entre los conjuntos residenciales modernos en las ciudades socialistas del Oeste y del Este de Europa, podemos sintetizar algunos diagnósticos y percepciones con el fin de determinar hasta qué punto estas formas urbanas de vivienda masiva pueden entenderse como el resultado de una cultura urbanística internacional moderna y responsables de la pérdida de urbanidad que se percibe en los conjuntos urbanos modernos de los años sesenta y setenta.

Si observamos los resultados desde la perspectiva de la morfología y del diseño urbano, enseguida percibimos que en el Este el número y tamaño de los conjuntos es bastante mayor que en el Oeste. Por otro lado, en los países del Este, a pesar de un cierto grado de experimentación y de la diversidad de formas urbanas, la mayoría de los conjuntos tienen un aspecto muy similar. A menudo sorprende la radical homogeneidad y el aspecto monolítico de los paisajes urbanos que los grandes conjuntos residenciales conforman en las ciudades socialistas del Este. Esto se debe más bien a su gran tamaño y a su monótona arquitectura que a su composición urbana; en particular, al fuerte compromiso con la estandarización y prefabricación (concretamente al uso de paneles modulares en las fachadas), pero también al código prevalente impuesto por la administración del Estado de acuerdo con la idea socialista de la uniformidad de las viviendas como símbolo de la igualdad de sus habitantes.

Una de las conclusiones más claras que se pueden extraer de esta visión comparada entre los conjuntos modernos del Este y del Oeste es que las diferencias que se aprecian en el diseño urbano no son tan relevantes entre los dos lados del Telón de Acero. Lo que sí es significativo es el contraste entre el nivel de experimentación o la diversidad de soluciones que se observa en los proyectos de la primera posguerra y el grado de estandarización y de radicalización de las soluciones, más simples y elementales, que caracteriza las intervenciones realizadas cuarenta años después de la formulación de los principios CIAM y que condujo a lo que podríamos llamar la vulgarización de la Carta de Atenas.

Esto significa que los problemas y las oportunidades derivados de esta forma urbana fueron bastante parecidos en ambos lados del Telón de Acero. Parece evidente que la creciente confianza en que la cultura urbanística y el urbanismo modernos ayudarían a promover una sociedad más justa fue el punto de partida tanto para técnicos como para arquitectos en las ciudades del Oeste y del Este de Europa. En los años sesenta, visiones más pragmáticas y la demanda de construcción rápida de viviendas derivó en el desarrollo de formas urbanas homogéneas casi en todas partes. ${ }^{62}$
Ciudad dormitorio modelo. Construida principalmente con bloques de paneles de hormigón, su mayor problema en la actualidad no son tanto los espacios públicos o el elaborado diseño urbano, cuanto la mala calidad de la construcción. Fuente: Wikimedia Commons y fotografía de los autores 2014.

[12] Sykhivs'kyi, Lviv, década de 1970. Este caso de estudio puede servirnos de comparación como ejemplo de cómo los grandes racion, como ejemplo decomo los grandes espacios públicos son a menudo un problema, especialmente cuando la construcción de los equipamientos se retrasa. Fuente: Bohdan Cherkes.

a 12.000 habitantes) se componen de 5 a 8 "super bloques" o complejos residenciales, cada uno con una población de entre 1.000 y 1.500 habitantes.

49 Goldzamt detalla estos principios: "Cada uno de estos distritos estaba pensado para albergar una población de 10.000 a 20.000 habitantes en un área entre 30 y 50 ha, con viviendas y servicios como tiendas, lavanderías, restaurantes, escuelas y equipamientos para niños, $y$ cada distrito debía estar conectado con el lugar de trabajo de sus habitantes." GOLZAMT, E., EI urbanismo en la Europa Socialista, op. cit.

50 "Ces colonies, cités, prototypes de la construction standarisée sont donc avant tout dans leurs fondements une expression de la modernité europeene, et non du "socialisme", Coudroy de Lille, L. "Une ideologie du préfabriqué?", en DUFAUX, F. Y FOURCAUT, A. (eds.), Le monde des grands ensembles, op. cit., pp. 90-91.

51 WAKEMAN, R.,"Rethinking postwar planning history", op. cit., pp. 153-163.

52 WARD, S., "Soviet communism and the British planning movement: rational learning or Utopian imagining?", Planning Perspectives, Vol. 27, 4, 2012

53 AMESTOY, I., "Les grands ensembles en Russie, de l'adoption dún modèle à la desáffection", en DUFAUX, F., FOURCAUT, A. (eds.), Le monde des grands ensembles, op. cit.

${ }^{54}$ Los grandes conjuntos urbanos de la posguerra en todos los países socialistas, de Rusia a Polonia, pasando por Alemania del Este albergan una parte significante de la población de las ciudades. COUDROY DE LILLE, L., ibídem.

${ }^{55}$ Los problemas vinculados con la prefabricación se evidencian sobre todo en los grandes conjuntos de los años sesenta. Criterios económicos determinaron la elección de diseños estándar para los grandes paneles y grandes bloques de edificios pertenecientes a la "primera generación" de conjuntos residenciales. Como afirma críticamente un autor como lkonnikov: "the minimal number of types of flats and apartment houses was adopted... The density of the construction was not high and the houses 'floated' freely in space, without organizing that space. All these circumstances taken together generated a drab monotony of the residential environment." IKONNIKOV, A., Russian Architecture of the Soviet Period, Moscú: Radugacop, 1988.

56 GOLDZAMT, E. El urbanismo en la Europa 
socialista, op. cit., pp. 262-263. En Praga, los nuevos conjuntos residenciales, ahora habitados por más de 500.000 personas, aproximadamente la mitad de la población aprimadamen conocen comelak (término coloquial utilizado para describir los edificios construidos con paneles de hormigó prefabricado y precomprimido, como los que se observan en la Antigua Checoslovaquia y en el antiguo bloque soviético).

${ }^{57}$ Conjunto proyectado para 150.000 habitantes desarrollado para el congreso de la UIA que tuvo lugar en Praga en 1967 y que fue presentado en el pabellón de checoslovaco de la exposición de Montreal de aquel año. También interesa tener en cuenta el concurso internacional para un proyecto de gran escala Petržalk, Bratislava (1967), que recibió 84 propuestas de 19 países diferentes; y también el distrito residencial Lazdynai construido en Vilnius, Lituania (1969), "el lugar más importante de Lituania para el urbanismo moderno en el que los arquitectos utilizaron modelos occidentales en lugar de la referencia a Moscú". GOLDZAMT, E., El urbanismo en la Europa socialista, op. cit., p. 316

${ }^{58}$ Construidos en la segunda mitad de los años ochenta y al inicio de los noventa en la parte norte de la ciudad. Bonice es un complejo de bloques prefabricados que aloja a más de 50.000 personas.

${ }^{59}$ Algunos datos pueden ilustrar este proceso: "From 1965 to 1973 the share of large-panel buildings in state-constructed and co-operatively constructed housing increased from $29 \%$ to $44 \%$, which, considering all housing production was an increase from 19\% to $31 \%$. By 1975 , about forty types of largepanel buildings had been developed. At the end of the 1970s the famous 5 and 12-storey series using pre-fabricated construction were introduced. The present high-rise type dominant in the Ukraine is the so-called multisection building with 5 or more sections of 5 or 9-storeys", DURMANOV, V., DUBBELING, D., Ukraine, Inheritance of centralized planning// High-rise housing in Europe, Delft: TU Delft, p. 206

${ }^{60}$ Proyectado por los arquitectos $\mathrm{G}$ Kulchitsky, V. Ladny, N. Pestryakov y los ingenieros A. Shtekel and V. Koval. Situado en la margen izquierda del río Dnieper, en un territorio desecado con arena. Estaba ideado como barrio sin coches privados, servido por autobuses y barcas que atravesaban el canal del Dnieper. Bloques entre 9 y 13 plantas en un área total de 130 ha, con 45.000 habitantes previstos en el proyecto y 27.400 resultantes en el censo de 2012. Informaciones proporcionadas por Ana Viazovska.

61 Una reciente visita (mayo de 2014) de los autores de este artículo resultó útil para confirmar algunos de estos argumentos.

62 Como resume WASSENBERG, F.: "There has never been a period in house building in which the similarities between countries have been as great. High-rise estates dominated the building in this era.", F. Wassenberg, Large Housing Estates, op cit. De hecho, muchos autores coinciden en que las similitudes fueron mayores en la década de los sesenta hasta mediados de los setenta, cuando "en y entre los paises del Norte y del Este de Europa se evidenciaron diferentes trayectorias." Ver TURKINGTON, R., VAN KEMPEN, Ver TURKINGTON, R., VAN KEMPEN,
R., WASSENBERG, F. (eds.), High-rise housing in Europe. Current trends and future prospects, Delft: Delft University Press, 2004, p. 5. Sin embargo, esto no significa que las diferencias no fueran relevantes.

63 La aparición, en el panorama internacional, de algunas redes como RESTATE (Restructuring Large Housing Estates in European Cities), cuyo objetivo es elaborar catálogos de buenas prácticas que ayuden a ofrecer nuevas visiones para intervenir en estos conjuntos, es una buena prueba de la actualidad y complejidad de un tema que requiere afrontar con lucidez y creatividad la ambivalencia del legado construido, intelectual y conceptual, de la Carta de Atenas.
Como algunos autores señalan, las mayores debilidades de estos conjuntos residenciales residen, fundamentalmente, en la negación radical de la calle y en la renuncia a las nuevas posibilidades que esta ofrecía; en la escala desmesurada y falta de definición de los espacios abiertos; y en la dificultad de su gestión y mantenimiento, ya que se consideran "tierra de nadie". Tras haber consultado fuentes secundarias y visitado un número significativo de conjuntos residenciales, tanto en el Oeste como en el Este, podemos decir que los aspectos más cuestionables -siempre desde la perspectiva del diseño urbano- son la rigidez, la enorme dimensión y falta de una clara definición formal de los espacios abiertos, así como la dificultad de su gestión y mantenimiento debido a su condición de "tierra de nadie". A pesar de la diversidad de las soluciones, no es fácil encontrar buenos ejemplos entre los conjuntos de gran tamaño, tanto en las ciudades del Este como en las de Oeste. Sin embargo, sí hemos encontrado bastantes ejemplos de buena urbanidad en los de tamaño medio, lo que confirma que la escala es un factor determinante del éxito de estos conjuntos.

Pero enfatizar los problemas y los errores de los conjuntos residenciales modernos (especialmente de los de gran escala) no significa ser "antimodernos" y tampoco criticar "la ciudad de las torres" de forma genérica, como es el caso de algunos autores. Lo que nos interesa es subrayar las debilidades de un modelo que a menudo ha terminado siendo la vulgarización de la Carta de Atenas, al aplicarse en grandes conjuntos y megaestructuras que casi siempre resultaron fallidas. A pesar de que existen diferentes versiones y algunos ejemplos interesantes, se trata tan solo de excepciones en un panorama determinado por proyectos de baja calidad que caracterizan casi todas las periferias de las ciudades europeas construidas entre los sesenta y los setenta.

Es necesario continuar investigando si queremos entender mejor las estrategias y los procesos que esconden las formas urbanas resultantes de estos conjuntos residenciales. Serían muy útiles algunos estudios sobre conexiones internacionales y difusión de conceptos, especialmente sobre el préstamo selectivo de ideas procedentes de múltiples fuentes, en la línea de los que están desarrollando algunos investigadores. Como ya hemos mencionado, este camino de difusión internacional y apropiación de ideas de diseño urbano se puede reconocer en algunos importantes proyectos de vivienda a gran escala en los países del Este que fueron objeto de exposiciones internacionales y de concursos en aquellos años.

Resulta interesante considerar el diagnóstico actual sobre esta tipología de conjuntos residenciales masivos elaborado por la Comisión Europea. Además de los problemas técnicos debidos a la baja eficiencia energética de los edificios y a los altos costes de mantenimiento, casi todos los conjuntos comparten los mismos problemas urbanos relacionados con la baja calidad y estado de conservación de los espacios públicos. Tanto en las ciudades de Este como en las del Oeste la gestión de los espacios públicos se ha convertido en un aspecto clave, además de la más reconocida necesidad de rehabilitación edificatoria. Aunque la recuperación es casi siempre necesaria, la cuestión es cómo proceder con este legado: por un lado, como legado construido de gran entidad cuantitativa; por otro, como legado disciplinar, (analizando hasta qué punto siguen resultando válidos los modelos "modernos" que ahora se siguen aplicando en contextos urbanos de rápido crecimiento como China o Corea)..$^{63}$

En cualquier caso, la validez internacional de los modelos también genera paradojas. El arte y la literatura muestran esta capacidad de las formas urbanas para servir a diferentes ideologías. Concluyamos con algunas citas al respecto que hacen referencia a las ironías y ambivalencias de la historia. Por ejemplo, la escritora alemana Brigitte Reimann escribe en su diario, al hablar de Berlín: "Tengo la sospecha de que la arquitectura moderna se parece en el Este y en el Oeste." ${ }^{64}$ También el escritor español Javier Pérez Andújar, refiriéndose a la periferia de Barcelona se expresa como sigue: "Un paisaje intercambiable con otras ciudades de todo el mundo (...) Antes de sentirme parte de un país, de una patria o de una nación, pertenezco a la internacional de los bloques." En una línea análoga, la artista eslovena Marjetica Potrc pone en evidencia la naturaleza utópica de los conjuntos residenciales modernos en "The Modernist Project: equality and justice for all... in the West and in the East."

\section{Agradecimientos}

Este artículo es una versión editada y ampliada de la comunicación presentada a la $12^{\text {th }}$ International Conference on Urban History Cities in Europe, Cities in the World, European Association for Urban History, Lisboa, Portugal, 3-6 septiembre $2014^{2}$. Los autores agradecen a Lydia and Laurent Coudroy de Lille así como a los revisores anónimos de rita_ sus comentarios. 